Natural Hazards and Earth System Sciences (2003) 3: 515-521

(C) European Geosciences Union 2003

Natural Hazards and Earth System Sciences

\title{
Slope stability monitoring from microseismic field using polarization methodology
}

\author{
Yu. I. Kolesnikov, M. M. Nemirovich-Danchenko, S. V. Goldin, and V. S. Seleznev \\ Institute of Geophysics SB RAS, 3 Pr. Akademika Koptyuga, 630090 Novosibirsk, Russia
}

Received: 7 October 2002 - Revised: 6 January 2003 - Accepted: 7 January 2003

\begin{abstract}
Numerical simulation of seismoacoustic emission (SAE) associated with fracturing in zones of shear stress concentration shows that SAE signals are polarized along the stress direction. The proposed polarization methodology for monitoring of slope stability makes use of three-component recording of the microseismic field on a slope in order to pick the signals of slope processes by filtering and polarization analysis. Slope activity is indicated by rather strong roughly horizontal polarization of the respective portion of the field in the direction of slope dip. The methodology was tested in microseismic observations on a landslide slope in the Northern Tien-Shan (Kyrgyzstan).
\end{abstract}

\section{Introduction}

Reliable assessment of slope stability, especially important in urban regions, helps to assure human safety, to cut down the costs and evaluate the efficiency of landslide mitigation, and to reduce economic losses from catastrophic landslides. Slope failure occurs when tangential stress overcomes the shear strength of rocks, and a rock mass detached from the slope moves downhill along a slip surface. The formation of a sliding surface is preceded by more or less long stress concentration in its vicinity associated with numerous microand medium-scale changes to strained rocks.

These changes include forming and healing of cracks accompanied by release of microseismic energy (called acoustic or seismoacoustic emission), which was observed in many experiments (Goodman and Blake, 1965; Cadman and Goodman, 1967; Postoev, 1972; Rouse et al., 1991). Seismoacoustic emission makes up a great portion of the microseismic field on active slopes, and its parameters (intensity, energy, and spectral patterns) have been broadly used in landslide monitoring (McCauley, 1976; Muravin et al., 1987; Muravin et al., 1991; Adushkin et al., 1993; Dixon et al., 1996).

Correspondence to: Yu. I. Kolesnikov

(kolesn@uiggm.nsc.ru)
Cracks that arise in the vicinity of a forming sliding surface often have preferred orientations produced by stresses in rocks, and polarization analysis appears a useful tool in studies of microseismic fields in landslide sites. First applications of this approach were reported by Zyatev et al. (1998) who revealed strongly differing polarization patterns of microseisms on active and inactive parts of slopes during landslide studies near Tomsk (West Siberia, Russia).

In this paper we apply numerical simulation to analyze the polarization of microseismic signals relevant to landslide hazard. The modeling results provided a basis for the polarization methodology to be used in slope stability monitoring from microseismic field. The methodology was tested during processing and interpretation of field data from threecomponent microseismic observations on a landslide slope in the Suusamyr valley in the Northern Tien-Shan, Kyrgyzstan.

\section{Numerical simulation of seismoacoustic emission from a forming sliding surface}

A landslide was modeled as a homogeneous isotropic parallelepiped with a square base (Fig. 1a). Its upper bound (the plane $Z=0$ ) is uniformly and constantly loaded along the $Y$-axis and the sides are free from loading. For the sake of computation, the study domain is represented as a grid of cubic elementary cells.

The bottom bound simulates the forming sliding surface. The weak zone is modeled under the assumption that at a zero time a half of the cells on the bottom bound can slide freely, without friction, along the $Y$-axis, and the other cells remain fixed in randomly set coordinates. This boundary condition, together with the conditions on the upper bound, provides stress concentration on the bottom bound.

It is assumed that a fixed cell may set into motion along the $Y$-axis as soon as a threshold stress is achieved ( $\sigma_{y z}$ component of stress tensor), i.e. a shear crack forms. The magnitude of critical stress is of no interest to our problem as it affects only the amplitude of the emitted signals. Thus the problem 


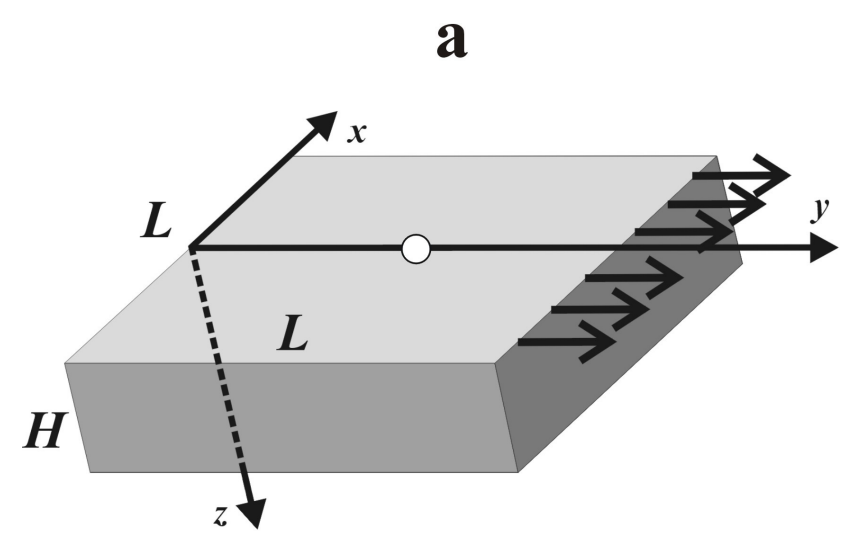

b
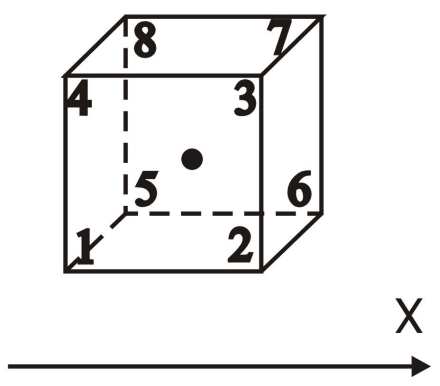

Fig. 1. Geometry of numerical model (a) and elementary cubic cell of computational grid (b).

is solved in a dynamic formulation with variable boundary conditions on the bound $Z=H$. The calculations are made using the first Cauchy's law (motion equations in stresses)

$\frac{\partial \sigma_{i j}}{\partial \zeta_{j}}=\rho \ddot{u}_{i}$

and the defining stress-strain relations as the incremental Hooke's law (Germain, 1962)

$\sigma_{i j}^{\nabla}=c_{i j k l} \dot{\varepsilon}_{k l}$,

where

$\sigma_{i j}^{\nabla}=\frac{d \sigma_{i j}}{d t}-\Omega_{i k} \sigma_{k j}-\Omega_{j k} \sigma_{k i}$

is Jaumann's derivative, $\sigma_{i j}$ and $\varepsilon_{i j}$ are stress and strain tensor components, respectively, $\Omega_{i j}$ are spin tensor components, $\rho$ is density, $u_{i}$ are components of displacement vector, $c_{i j k l}$ are components of fourth-order tensor of elastic constants, $t$ is time, and $\zeta_{i}$ are Cartesian coordinates $X, Y$, and $Z$.

Displacements and particle velocities are found in the nodes of the cubic cells, and strain velocities are calculated from displacement velocities in their centers:

$\dot{\varepsilon}_{i j}=\frac{1}{2}\left(\frac{\partial \dot{u}_{i}}{\partial \zeta_{j}}+\frac{\partial \dot{u}_{j}}{\partial \zeta_{i}}\right)$

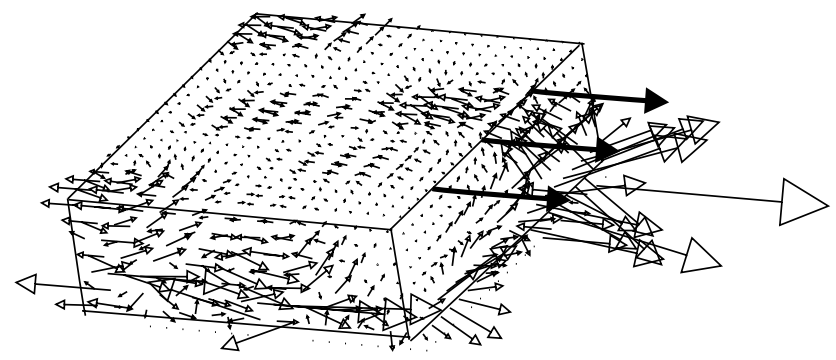

Fig. 2. Computed displacement velocities (vectors) for the central part of the model. Arrows show direction of load applied to upper bound.

Spatial derivatives are obtained by averaging over the respective bounds of the cubic cells, e.g. for the $\zeta_{11}$ component it is (indexing of nodes is in Fig. 1b)

$\dot{\varepsilon}_{11}=\frac{1}{4 \Delta \zeta}\left(\left(\dot{u}_{1}\right)_{2}+\left(\dot{u}_{1}\right)_{3}+\right.$

$\left.\left(\dot{u}_{1}\right)_{6}+\left(\dot{u}_{1}\right)_{7}-\left(\dot{u}_{1}\right)_{1}-\left(\dot{u}_{1}\right)_{4}-\left(\dot{u}_{1}\right)_{5}-\left(\dot{u}_{1}\right)_{8}\right)$,

where $\Delta \zeta$ is side of cells.

Other components are determined in the same way. For details of the method see Nemirovich-Danchenko (1998). The method allows simulation of SAE signals associated with the formation of a sliding surface. The modeling parameters we used were $H=20 \mathrm{~m}$ for the thickness of the parallelepiped, $L=200 \mathrm{~m}$ for the length and width of its base, $P$-wave velocity $V_{P}=1000 \mathrm{~m} / \mathrm{s}, S$-wave velocity $V_{S}=500 \mathrm{~m} / \mathrm{s}$, density $\rho=2200 \mathrm{~kg} / \mathrm{m}^{3}$, and a $0.5 \mathrm{~m}$ grid (side of cells).

The modeling shows that loading applied to the upper bound causes stress concentration on the bottom bound. A shear crack arises when the critical stress is achieved in any computation cell. The forming of shear cracks, in turn, releases elastic energy producing sources of seismic waves distributed randomly over the model base.

Figure 2 shows a computed vectorial wave pattern for the central part of the model $\left(40 \times 40 \times 20 \mathrm{~m}^{3}\right)$ for the doubled traveltime of a direct wave from the earliest triggered source, i.e. the effect of the lateral model boundaries is excluded. SAE signals from several events are clearly seen in the figure, which is actually a numerical snapshot of the vector field of displacement velocities. The maximum-amplitude vectors on the upper bound of the model for each event roughly follow the direction of the load applied to this bound.

A better idea of the polarization of SAE signals on the upper bound of the model can be obtained from computation results for three displacement velocity components in the point $(0, L / 2,0)$ on this surface (white circle in Fig. 1a). Figure 3 shows example polarization curves based on these results for the planes $(X, Y)$ and $(Y, Z)$, which represent particle motion in the model medium on the surface $Z=0$. As we expected, the polarization of signals roughly corresponds to the direction of the $Y$-axis. 


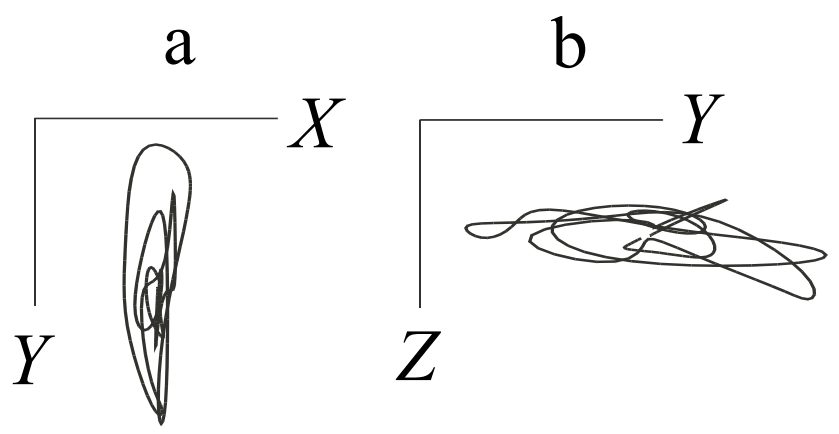

Fig. 3. Computed projections of particle trajectories on planes $Z=$ 0 (a) and $X=0$ (b).

Note that the trajectory shown in Fig. 3 is a stack of signals from several sources in different points of the model's lower bound, i.e. the direction of predominant polarization on the surface depends rather on the direction of shear stress than on the position of the sources. Applied to slope processes (on relatively shallow slopes), this is a roughly horizontal polarization of a certain portion of the microseismic field along the slope dip. Isolated from the total field, this portion may be diagnostic of slope activity.

\section{Field microseismic observations on a landslide slope}

The polarization methodology was tested during processing of field data from microseismic observations on a landslide slope in the Suusamyr valley (Northern Tien-Shan, Kyrgyzs$\tan$ ). The landslide (Fig. 4), 250-300 $\mathrm{m}$ in width and in length, was produced by the $M=7.3$ Suusamyr earthquake of August 1992 at an altitude of $2400 \mathrm{~m}$. The displaced rocks are up to $50-70 \mathrm{~m}$ thick and estimates of their volume are from 0.5 to $1.0 \times 10^{6} \mathrm{~m}^{3}$ (Havenith et al., 2000). The slope in this site is about $30^{\circ}$ steep and dips towards an azimuth of $140-160^{\circ}$. It is composed of Quaternary sands and clays lying upon consolidated clays and includes bedrock blocks. In its lower part the landslide splits into three lobes (each a few hundreds of meters long) separated by unbroken parts of the slope.

The latter are also potentially hazardous as they are cut with numerous surface cracks, up to tens of centimeters wide. Some of them are accompanied by micro-landslides. The microseismic field in various parts of the slope (Fig. 4c) was monitored by a portable four-channel digital station based on a laptop computer. The instrument, including a battery, cables, a three-component geophone, and pre-amplifiers, is mounted on a small two-wheel carriage easy to draw even in the mountains.

The instrument has a broad frequency band from $10 \mathrm{~Hz}$ to $100 \mathrm{kHz}$ with programmable digitization frequency from $2 \mathrm{kHz}$ to $1 \mathrm{MHz}$. The broadband recording was used, as we had no a priori information on the frequency content of the microseismic field in this region. According to published data (Muravin et al., 1991), the frequency of SAE signals
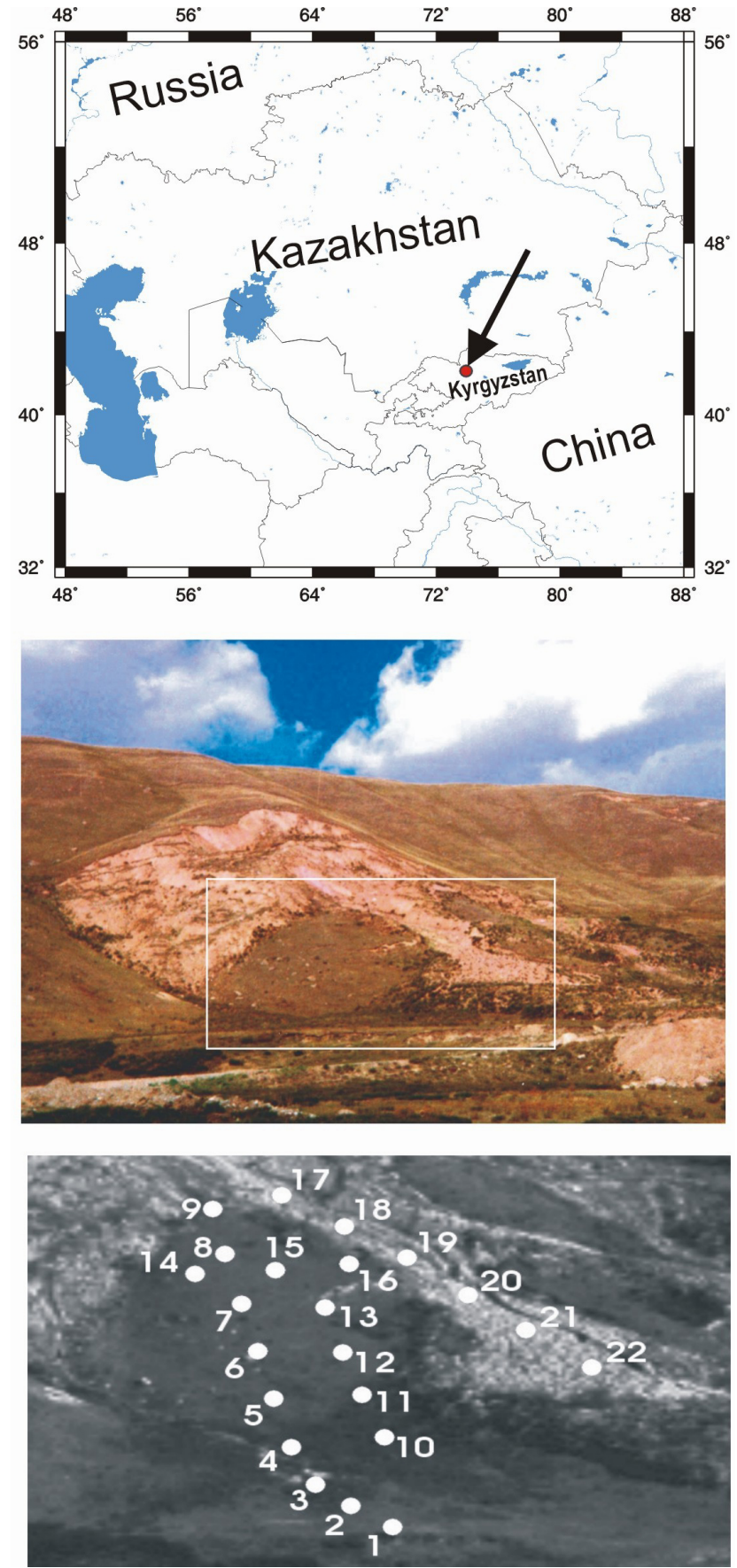

Fig. 4. (a) Location map of Kyrgyzstan (landslide site is shown by arrow). (b) Landslide slope and monitoring area (within the box). (c) Instrument layout.

recorded on slopes prone to landsliding may vary from a few hertz to hundreds of kilohertz in function of slope geology.

After preliminary acquisition we selected the bandwidth that encompassed the greatest portion of microseismic energy and continued the experiments in a range of 10 to $500 \mathrm{~Hz}$ with a three-component geophone consisting of three SM 4/7 sensors inside one case, oriented to the south ( $X$ component), to the east ( $Y$ component), and upwards $(Z$ 


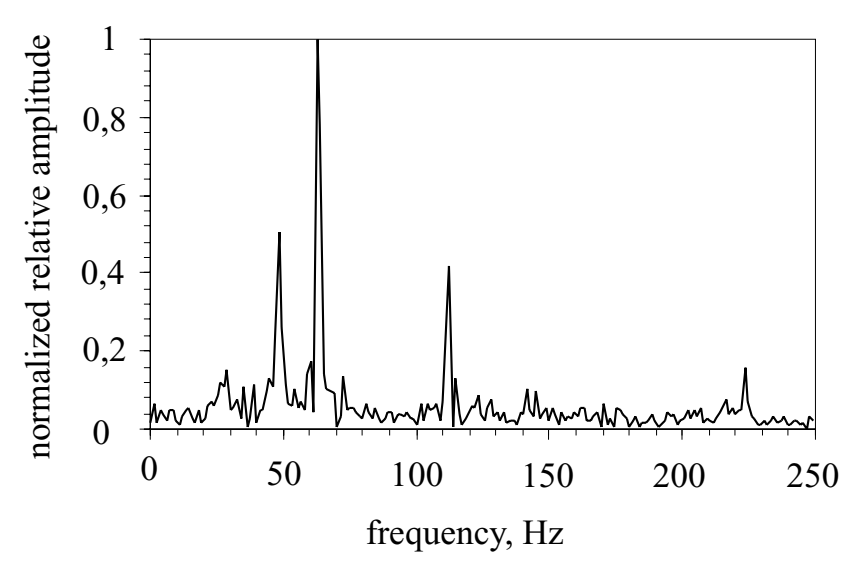

Fig. 5. Spectrum of a non-filtered microseismic signal.

component). At each measurement point five $0.6 \mathrm{~s}$ time series were acquired and digitized at a step of $0.1 \mathrm{~ms}$.

\section{Processing and analysis of microseismic data}

The observations were run in noisy conditions because of roadwork in the vicinity of the landslide, which required a preliminary spectral analysis of the records. The spectra of most traces contained typically cultural quasi-harmonic noise in the $50-120 \mathrm{~Hz}$ bandwidth and above $210 \mathrm{~Hz}$, standing out as narrow peaks about 50,65, 110, and $225 \mathrm{~Hz}$ (Fig. 5). Therefore, we applied 10-45 and 120-210 Hz bandpass filtering and used two sets of filtered data in the further processing.

The next step implied visualization of the projections of particle motion onto horizontal and vertical planes. The polarization of the projections showed no regularity in the broad band (prior to filtering, Fig. 6a) and at low frequencies (10$45 \mathrm{~Hz}$, Fig. 6b), but a well-defined polarization was observed in the high-frequency bandwidth (120-210 Hz, Fig. 6c). Polarization had about the same direction as the slope dip, except for few weakly polarized responses on a terrace beneath the slope, on rocks over large cracks, and on the surface of rocks deformed by the landslide, i.e. in unloaded points.

The polarization patterns at different frequencies may be explained as follows. The low-frequency signals, which make up the greatest portion of the broadband (non-filtered) response, come from remote sources outside the landslide slope and thus have no preferred orientations.

The signals from remote high-frequency sources, which are as a rule weaker than the low-frequency ones, almost elude recording because of spreading and attenuation. Therefore, polarization of high-frequency noise may be associated with the forming of micro-cracks in rocks, which is implicitly confirmed by the orientation of the principal polarization axis along the slope dip.

Then we determined the numerical parameters of polarization by more detailed processing of the horizontal and vertical projections of particle motion from the high-frequency
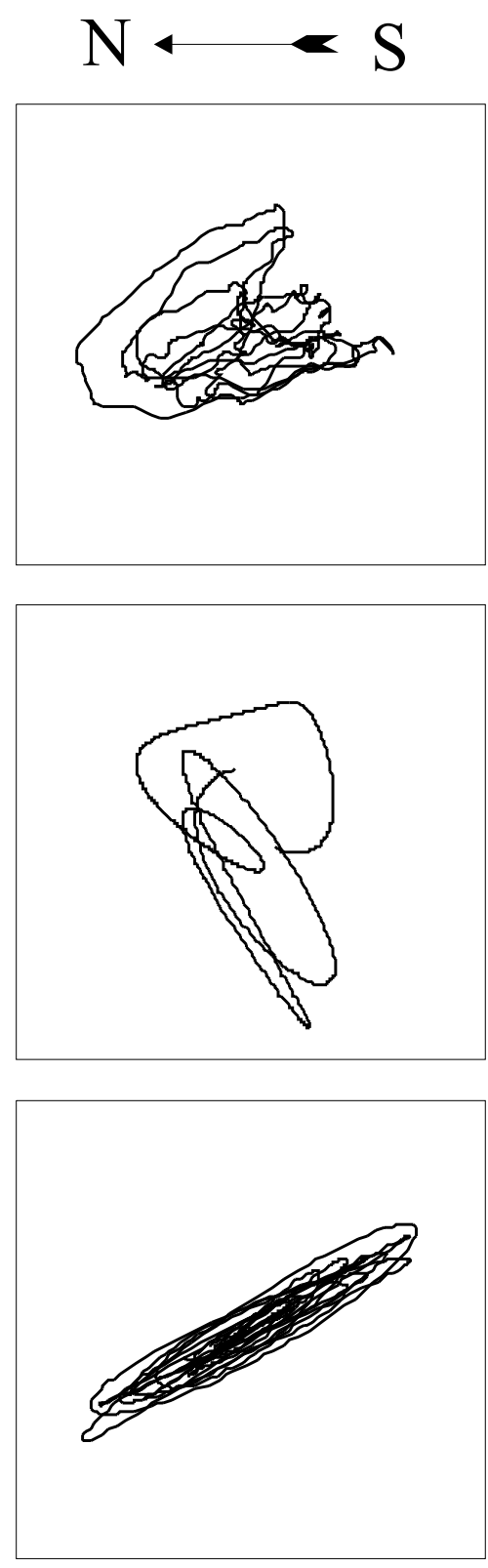

Fig. 6. Horizontal projections of particle trajectories in a point on slope, from records before (a) and after $10-45 \mathrm{~Hz}$ (b) and 120 $210 \mathrm{~Hz}$ (c) bandpass filtering.

portion of the microseismic field, using five $0.6 \mathrm{~s}$ threecomponent records from each point.

The seismic traces $x_{i}, y_{i}, z_{i}(i=1, \ldots, N)$ recorded by the three-component geophone with its sensors oriented along the coordinates $X, Y, Z$, were considered as sets of the respective coordinates of the geophone subject to discrete variations with time under the effect of microseisms. We applied least-square approximation by a straight line $y=a x$ to the points $\left(x_{i}, y_{i}\right)$ on the horizontal plane $Z=0$, where $a$ is given by

$a_{1,2}=\frac{-b \pm \sqrt{b^{2}+4 c^{2}}}{2 c}$, 
where

$b=\sum_{i=1}^{N}\left(x_{i}^{2}-y_{i}^{2}\right)$,

$c=\sum_{i=1}^{N} x_{i} y_{i}$

Then standard deviations $\sigma_{1}$ and $\sigma_{2}$ of the points $\left(x_{i}, y_{i}\right)$ from the lines $y=a_{1} x$ and $y=a_{2} x$ (one approximating the set of points and the other positioned perpendicular to it) were found as

$\sigma_{1}=\sqrt{\frac{\sum_{i=1}^{N}\left(x_{i} \sin \alpha_{1}-y_{i} \cos \alpha_{1}\right)^{2}}{N}}$,

$\sigma_{2}=\sqrt{\frac{\sum_{i=1}^{N}\left(x_{i} \sin \alpha_{2}-y_{i} \cos \alpha_{2}\right)^{2}}{N}}$,

where

$\alpha_{1}=\tan ^{-1} a_{1}$,

$\alpha_{2}=\tan ^{-1} a_{2}$

Finally, the polarization factor in the horizontal plane was expressed through the parameter $P_{h}$ calculated from the standard deviations $\sigma_{1}$ and $\sigma_{2}$ as

$P_{h}=1-\frac{\min \left(\sigma_{1}, \sigma_{2}\right)}{\max \left(\sigma_{1}, \sigma_{2}\right)}, \quad\left(0 \leq P_{h} \leq 1\right)$.

The parameter $P_{h}$ is a numerical expression of polarization in some direction within the plane $Z=0$. The direction is defined by a straight line drawn through the origin of the coordinates at an angle $\alpha_{h}$ (polarization angle) to $X$, which is either $\alpha_{1}$ or $\alpha_{2}$, depending on which of the two corresponds to the least standard deviation of the points $\left(x_{i}, y_{i}\right)$ from the respective straight line $y=a x$.

This line controls the position of the vertical plane, which was the subject of the further processing. The parameters of polarization in the vertical plane (polarization factor $P_{v}$ and angle $\alpha_{v}$ ) were found in the same way as for the horizontal plane, but the trace $z_{i}$ was used instead of $y_{i}$ and the projection $\xi_{i}$ onto the horizontal polarization direction instead of $x_{i}$, where $\xi_{i}$ is given by

$\xi_{i}=x_{i} \cos \alpha_{h}+y_{i} \sin \alpha_{h}$.

Thus, the polarization of high-frequency microseismic noise can be analyzed using four parameters - the polarization factors $P_{h}$ and $P_{v}$, and the angles $\alpha_{h}$ and $\alpha_{v}-$ that represent polarization in a horizontal and a vertical planes, respectively (Table 1). Note that we limited ourselves to just one possible approach to polarization analysis. Similar results can be obtained from other algorithms, such as the covariance matrix approach (Flinn, 1965).

\section{Discussion}

Now we interpret the processed field data (Table 1) with regard to numerical modeling (Sect. 2). The modeling shows that SAE signals from the zone of maximum shear stress should polarize mostly along the stress direction. The polarization of the fracturing-related portion of the microseismic field on relatively shallow-dipping slopes is nearly horizontal and dip-parallel.

Of course, this polarization pattern may be distorted by refractions and reflections in the landslide body producing a complex velocity structure (e.g. numerous steep interfaces). However, earlier studies of the same slope by active seismic and electric techniques (Havenith et al., 2000) show a quite plain upper section.

We analyzed the polarization of particle motions caused by microseisms in the $120-210 \mathrm{~Hz}$ bandwidth (the bandwidth hypothesized to represent seismoacoustic emission associated with fracturing in zones of stress concentration).

The signals from the river terrace (points 1-3) sloping at $\leq 10^{\circ}$ show a weak horizontal polarization in a direction roughly parallel to the slope dip, with low polarization factors. The factors of vertical polarization are also low, i.e. horizontal and vertical oscillations have comparable amplitudes. The weak polarization indicates that the SAE component is much below the microseisms from other sources (wind, water streams, or cultural noise, etc.), which allows us to assume that the slope within the terrace is inactive. The records on the surface of rocks deformed by landsliding (points 17-22) have small vertical components, i.e. show a nearly horizontal polarization. Polarization in the horizontal plane is rather weak in three points in the middle of the profile (points 18-20) but very strong in the upper and two lower points, where the oscillations are polarized roughly along the slope dip. This part of the landslide is U-shaped following a small gully made by a creek originated from a groundwater spring in the upper point of the profile, along the central lobe, and this polarization pattern may have been produced by effects of secondary slopes.

In the lower part of the profile (points 21-22) the surface flattens as the lobe reaches the river terrace, and the slope is much less dissected. The ground is wetter there because of poor consolidation and infiltration from the creek running downhill (the infiltration is implicitly indicated by small water basins in the creek vicinity). The water-saturated ground loses its strength, which may increase polarization in this part of the profile. The uppermost point on the lobe (point 17) also shows a strong polarization apparently associated with additional loading as $\sim 20 \mathrm{~m}$ uphill from the station the slope abruptly becomes much steeper making a $10 \mathrm{~m}$ high scarp.

The signals from rocks not involved into landsliding (between the central and left lobes, points 4-16) are in general highly polarized, both in the horizontal and vertical planes: nearly horizontally in the vertical plane and mostly dip-parallel in the horizontal plane. The high $P$ factors may attest to crack growth, which is confirmed by the fact that 
Table 1. Parameters of polarization of microseismic field on landslide slope within $120-210 \mathrm{~Hz}$ bandwidth

\begin{tabular}{|c|c|c|c|c|c|c|}
\hline $\begin{array}{l}\text { Number } \\
\text { of } \\
\text { station }\end{array}$ & $\begin{array}{c}\text { Location of } \\
\text { station }\end{array}$ & $\begin{array}{c}\text { Polarization } \\
\text { factor } P_{h} \text { in } \\
\text { horizontal } \\
\text { plane }\end{array}$ & $\begin{array}{c}\text { Polarization } \\
\text { angle } \alpha_{h} \text { in } \\
\text { horizontal } \\
\text { plane }^{a}, \text { deg }\end{array}$ & $\begin{array}{c}\text { Polarization } \\
\text { factor } P_{v} \text { in } \\
\text { vertical } \\
\text { plane }\end{array}$ & $\begin{array}{c}\text { Polarization } \\
\text { angle } \alpha_{v} \text { in } \\
\text { vertical } \\
\text { plance }^{b} \text {, deg }\end{array}$ & Remark \\
\hline 1 & river & 0,45 & $26(154)$ & 0,13 & 49 & - \\
\hline 2 & terrace & 0,5 & $-2(182)$ & 0,38 & 81 & - \\
\hline 3 & & 0,37 & 37 (143) & 0,14 & 36 & - \\
\hline 4 & unbroken & 0,4 & $-47(227)$ & 0,44 & 27 & lower point \\
\hline 5 & slope & 0,78 & $23(157)$ & 0,7 & 9 & - \\
\hline 6 & & 0,67 & $36(144)$ & 0,75 & 6 & - \\
\hline 7 & & 0,3 & $-46(226)$ & 0,51 & 29 & over a crack \\
\hline 8 & & 0,73 & 31 (149) & 0,81 & 3 & - \\
\hline 9 & & 0,55 & $30(150)$ & 0,37 & 28 & upper point \\
\hline 10 & & 0,7 & 35 (145) & 0,74 & 4 & - \\
\hline 11 & & 0,47 & 23 (157) & 0,56 & 13 & - \\
\hline 12 & & 0,65 & 33 (147) & 0,65 & 7 & - \\
\hline 13 & & 0,88 & $10(170)$ & 0,89 & 3 & - \\
\hline 14 & & 0,69 & $-1(181)$ & 0,56 & 22 & - \\
\hline 15 & & 0,77 & 31 (149) & - & - & - \\
\hline 16 & & 0,07 & 46 (134) & - & - & over a crack \\
\hline 17 & deformed & 0,84 & $20(160)$ & 0,5 & -4 & beneath the scarp \\
\hline 18 & rocks & 0,32 & $-11(191)$ & 0,63 & 9 & - \\
\hline 19 & (landslide & 0,3 & $-6(186)$ & 0,35 & -8 & - \\
\hline 20 & body) & 0,17 & $-3(183)$ & 0,7 & -1 & - \\
\hline 21 & & 0,59 & 32 (148) & 0,41 & -8 & wet ground \\
\hline 22 & & 0,66 & $20(160)$ & 0,79 & -12 & wet ground \\
\hline
\end{tabular}

${ }^{a}$ Counter-clockwise from southward direction. Numbers in parentheses are angles expressed as azimuth (dip azimuth of slope in monitoring site is about $140-160^{\circ}$ ).

${ }^{b}$ Angles relative to the horizontal plane, positive upward and negative downward facing the terrace.

polarization is weak or absent over large cracks (points 7 and 16 ), i.e. in points of unloading.

Polarization is also weak in the lower part of the unbroken slope (point 4) where the ground is naturally confined by the terrace below, and in its uppermost part (point 9) above which the slope abruptly becomes much lower-angle. In general, the observed relatively strong polarization of the highfrequency portion of the microseismic response from unbroken parts of the slope may indicate potential landslide hazard. Landslide may be triggered by seismic or hydrological and meteorological effects.

\section{Conclusions}

The numerical simulation showed that fracturing in zones of shear stress concentration may cause polarization of SAE signals along the stress direction. This property of the SAE portion of the microseismic field made the basis of the proposed polarization methodology applicable to the monitoring of slope stability. The method implies passive threecomponent recording of the microseismic field on a slope and picking its portion related to slope processes by filtering and polarization analysis. The activity of the slope is indicated by strong horizontal polarization parallel to the slope dip in the proper frequency components. This methodology was proven to be efficient during processing of field data from a landslide site in the Northern Tien-Shan and can be successfully used, along with other methods, for slope stability monitoring in regions of landslide hazard.

Acknowledgements. The study was supported by grants IC15CT97-0202 (EC, DG XII) from the European Community and 0005-65337 from the Russian Foundation for Basic Research. We thank A. D. Duchkov, A. F. Emanov, and T. A. Charimov for organization and S. V. Polozov and G. V. Larkin for logistic support of the field experiments. The paper profited much from the constructive criticism of two anonymous reviewers.

\section{References}

Adushkin, V. V., Spivak, A. A., Bashilov, I. P., Spungin, V. G., Dubinya, V. A., and Ferapontova, E. N.: Relaxation monitoring in the Southern Alps on unstable mountain slopes, Izv. AN SSSR, Ser. Fizika Zemli, (in Russian), 10, 103-107, 1993.

Cadman, J. D. and Goodman, R. E.: Landslide noise, Science, 158, 3805, 1182-1184, 1967. 
Dixon, N., Kavanagh, J., and Hill, R.: Monitoring landslide activity and hazard by acoustic emission, J. Geol. Soc. China, 39, 4, 437484, 1996.

Flinn, E. A.: Signal analysis using rectilinearity and direction of particle motion, Proc. IEEE, 53, 12, 1874-1876, 1965.

Germain, P.: Mécanique des milieux continus, Paris, 1962.

Goodman, R. and Blake, W.: An investigation of rock noise in landslides and cut slopes, Rock Mechanics and Engineering Geology, 2, 88-93, 1965.

Havenith, H.-B., Jongmans, D., Abdrakhmatov, K., Trefois, P., Delvaux, D., and Torgoev, I. A.: Geophysical investigations of seismically induced surface effects: case study of a landslide in the Suusamyr Valley, Kyrgyzstan, Surv. Geophys., 21, 4, 349-369, 2000.

McCauley, M. L.: Microseismic detection of landslides, In: Innovation in Subsurface Exploration of Soil, National Research Council (National Academy of Sciences), Transport Research Board, 581, 25-30, 1976.

Muravin, G. B., Erminson, A. L., and Sigalovsky M. N.: A system for investigation of ground motion by acoustic emission, Defektoskopia, (in Russian), 3, 62-66, 1987.

Muravin, G. B., Sigalovsky M. N., Rozumovich, E. E., and Lezvinskaya L. M.: Acoustic emission applied to ground motion studies (An overview), Defektoskopia, (in Russian), 11, 3-17, 1991.

Nemirovich-Danchenko, M. M.: A model for the brittle hypoelastic medium: the application to computation of deformations and failure in rock, Physical Mesomechanics, 2, 101-108, 1998.

Postoev, G. P.: Some results of seismoacoustic monitoring in a landslide site in the Zeravshan valley, Bull. VSEGINGEO, (in Russian), Moscow, 56, 65-71, 1972.

Rouse, C., Styles, P., and Wilson, S. A.: Microseismic emissions from flowslide-type movements in South Wales, Eng. Geol., 31, 1, 91-110, 1991.

Zyatev, G. G., Merkulov, V. P., and Nikolsky, A. A.: The reflection of landslip processes in geophysical fields, 60th EAGE Conf. and Techn. Exhib., Leipzig, 8-12 June, 1998: Oral and Poster Present., Geophys. Div., Extend. Abstr. Book, Leipzig, 121, 1998. 